\title{
Las competencias profesionales del nutricionista deportivo
}

\author{
The professional competences of \\ the sports dietitian
}

Maria Luisa BELLOTTO'

Imma Palma LINARES²

\section{R E S U M E N}

\section{Objetivo}

El artículo presenta los resultados obtenidos en la investigación que dio origen a la tesis doctoral defendida por la autora en la Universitat de Lleida (España), cuyo objetivo fue identificar las Competencias Profesionales de los nutricionistas que trabajan en el ámbito de la Nutrición Deportiva.

\section{Métodos}

Fueron investigados 14 expertos provenientes de Australia $(n=1)$, Brasil $(n=7)$, España $(n=3)$ y Estados Unidos $(n=3)$. La herramienta metodológica utilizada fue la técnica Delphi, compuesta de tres rondas de cuestionarios. En la primera ronda los expertos proporcionaron, a través de sus discursos, la identificación de un listado de Competencias Profesionales, información que en la segunda y tercera ronda pudieron ser evaluadas y posteriormente analizadas a través de cálculos estadísticos descriptivos (media, moda, mediana y desviación Standard).

\section{Resultados}

De esta manera, se llegó al consenso entre los expertos sobre 147 competencias profesionales identificadas. Las competencias fueron clasificadas en cuatro macro categorías de Competencias Profesionales: Competencias Técnicas (38), Metodológicas (62), Participativas (24) y Personales (23).

\section{Conclusión}

Los resultados demostraron que el estudio sistematizado de las Competencias Profesionales del Nutricionista Deportivo contribuye para el establecimiento de los contenidos que deben componer la disciplina de Nutrición Deportiva a ser incorporada en los itinerarios curriculares de las carreras de Nutrición Humana y Dietética.

Términos de indexación: Deportes. Nutricionista. Competencia profesional. Curriculum.

\footnotetext{
1 Faculdade Metropolitana de Campinas, Integrante do Grupo de Estudos de Nutriçao Aplicada a Atividade Física, Departamento de Ciencias do Esporte. R. Abolição, 1827, Bairro Swift, Campinas, SP, Brasil. Correspondência para/Correspondence to: M.L. BELLOTTO.E-mail:<malubs@hotmail.com>.

2 Universitat de Barcelona, Faculdad de Farmácia, Departamento de Nutrición y Bromatologia. Barcelona, España.
} 


\section{A B S T R A C T}

\section{Objective}

This article presents the results of the doctoral research developed by the author at the University of Lleida (Spain) in June 2006. The main goal was to investigate the professional competences of dietitians who work in the field of Sports Nutrition.

\section{Methods}

Fourteen experts in Sports Nutrition from 4 countries were investigated, Australia $(n=1)$, Brazil $(n=7)$, Spain $(n=3)$ and the United States $(n=3)$. The methodological tool applied was the Delphi Technique, consisting of three rounds of questionnaires. In the first round, the experts identified a number of Professional Competences, and on the following second and third rounds these competences were assessed and analyzed by descriptive statistics (mean, mode, median and standard deviation).

\section{Results}

A consensus was reached by the experts on 147 identified competences. The competences were classified into four macro categories of professional competences: Technical (38), Methodological (62), Participative (24) and Personal (23) competences.

\section{Conclusion}

The results showed that the systemized study of the professional competences of the Sports Dietitian contributes to the establishment of the contents to be taught in Sports Nutrition classes that are now being incorporated in the curriculum of undergraduate nutrition courses.

Indexing terms: Sports. Nutricionist. Professional Competence. Curriculum.

\section{INTRODUCCIÓN}

La literatura científica señala que el profesional especializado en Nutrición Humana y Dietética se ocupa de los diversos aspectos que envuelve la alimentación y la nutrición humana, tales como: la evaluación del estado nutricional, la educación alimentaria y nutricional de las personas, la gestión de centros de alimentación colectiva, el desarrollo de investigaciones de base y su aplicación en áreas como la dietoterapia, la genética, el metabolismo, la nutrición preventiva, entre otros ${ }^{1}$. Esto indica que dicho profesional puede actuar en diferentes ámbitos de la nutrición, como es la Nutrición Clínica, la Salud Pública, la Restauración, las Consultas Privadas, en el Marketing de productos alimenticios, en el ámbito de la Docencia, en las Asesorías y Consultorías, etc.

La evolución de la ciencia de la nutrición generó nuevas salidas profesionales y nuevas Competencias Profesionales para los Nutricionistas, evidenciando la necesidad de actualización de los planes de estudios o programa curricular de las carreras de grado superior de Nutrición Humana y Dietética. Según la American Dietetic Association (ADA) ${ }^{1}$, a lo largo de las últimas décadas, varios estudios han detectado la emergencia de nuevas Competencias Profesionales del Nutricionista sin que se hayan producido modificaciones en el modelo educacional básico impartido por las universidades desde 1927. La formación actual parece, en muchos casos, estar obsoleta y ser incapaz de atender a todas las necesidades actuales de esta profesión, especialmente en las áreas emergentes y por lo tanto novedosas.

En un ámbito de actuación en particular, como es la Nutrición Deportiva, el Nutricionista es cada vez más solicitado para atender a los deportistas y a las personas que practican habitualmente ejercicio físico y que quieren, a través de una conducta alimentaria adecuada, mantener un buen estado de salud y optimizar su rendimiento ${ }^{2-3}$. No obstante, Clark $^{4}$ alerta que "hoy día no existe una padronización de la práctica profesional para los nutricionista que quieran actuar en el área de la Nutrición Deportiva. Los nutri- 
cionistas y los alumnos de nutrición que quieran especializarse en nutrición deportiva sufren por la falta de orientación profesional y educacional". Así mismo, la actuación y las investigaciones en este sector han aumentado significativamente en las últimas dos décadas ${ }^{4-9}$.

En un estudio realizado previamente ${ }^{10}$ se constató que los programas curriculares de Nutrición Humana y Dietética de diferentes universidades brasileñas incluyen temas pertinentes a la Nutrición Deportiva de manera superficial y en su mayoría, definidos como contenidos complementarios de otras disciplinas de la nutrición, ofreciendo, de este modo, una formación escasa y en ocasiones insuficiente, lo que obliga a los interesados a realizar estudios de postgrado complementarios. Aunque algunas universidades empezaron a impartir esta disciplina de forma independiente (en muchos casos como asignatura optativa), sus contenidos fueron establecidos según los criterios adoptados por cada institución en particular ${ }^{11}$, lo que puede generar dificultades de diálogo entre los profesionales provenientes de distintos centros formativos.

En la opinión de Le Boterf12, "las necesidades de formación no existen de por sí, sino que constituyen desviaciones que es preciso identificar y analizar en relación con las situaciones concretas o las referencias que se encuentran en su origen (disfunciones, proyectos, evolución de las profesiones y de los empleos, evoluciones culturales...". Por este motivo, se creyó necesario definir y describir de manera rigurosa y sistematizada cuales conocimientos técnicos, sociales, culturales, pedagógicos, profesionales, etc., componen las Competencias Profesionales específicas del Nutricionista Deportivo.

A raíz de analizar los puntos anteriormente citados, se planteó como principal objetivo de estudio de esta investigación el desvelar cuales deben ser las Competencias Profesionales del Nutricionista Deportivo, mediante un estudio descriptivo basado en el discurso de profesionales expertos que ejercen esta profesión ${ }^{13}$.

\section{Las competencias profesionales, las ciencias de la nutrición y la nutrición deportiva}

El concepto de Competencias Profesionales está asociado al análisis de las actividades profesionales y al inventario de lo que es necesario para que un profesional pueda desarrollar adecuadamente su misión ${ }^{14}$. Para esta investigación optamos por la definición establecida por Bunk ${ }^{15}$, que resume el significado de competencia: "El individuo que posee Competencia Profesional dispone de los conocimientos, las destrezas y las actitudes necesarias para ejercer su propia actividad laboral, resuelve los problemas de forma autónoma y creativa y está capacitado para colaborar en su entorno laboral y en la organización del trabajo".

A partir del momento, en que los programas educacionales empezaron a basarse en las Competencias Profesionales propias de los profesionales, fue posible desvelar y propiciar los cambios necesarios en el sistema de salud, acercándose, de esta forma, a las demandas del mercado laboral y a la mejoría de la educación universitaria para los futuros profesionales ${ }^{16}$. Para la Nutrición Deportiva desvelar sus Competencias Profesionales es fundamental la adecuación de la formación del mismo. "La Nutrición esta desarrollándose". Así como muchas carreras de los años 90, la clave para la supervivencia es el reconocimiento de sus competencias tronchales y la determinación de cómo alcanzar estas competencias a través de la carrera de nutrición y de programas de educación continuada"17.

El conocimiento de las Competencias Profesionales permite solucionar problemas futuros y conflictos del mercado laboral, además de preparar mejor a las personas para la vida y para la labor cotidiana ${ }^{18}$. La necesidad de establecer las Competencias Profesionales específicas de cada área de actuación del nutricionista, es una responsabilidad que debe ser asumida por las instituciones responsables de la formación de dicho profesional ${ }^{19-20}$. Es por ello, que poner al descubierto 
las Competencias Profesionales del Nutricionista Deportivo, es de gran relevancia para establecer qué contenidos deben ser abordados por una disciplina específica de Nutrición Deportiva en la carrera universitaria de Nutrición. Esta disciplina debe pretender garantizar una adecuada formación de las futuras generaciones de profesionales en este ámbito.

\section{MÉTODOS}

El modelo analítico - conceptual de esta investigación se basa en el paradigma cualitativo con el intuito de descubrir, captar y comprender una teoría, una explicación y un significado ${ }^{21}$ a partir del discurso de profesionales expertos en el área de Nutrición Deportiva. A pesar de la técnica de investigación aplicada a esta investigación ser de naturaleza cualitativa, el tratamiento de los datos también pudo ser realizado de manera cuantitativa dependiendo de la fase de la investigación. No obstante, para el análisis y la interpretación final de los datos se utilizaron técnicas cualitativas. Esta investigación también es considerada analítica, por tratar de investigar la problemática de una categoría de profesionales y sus respectivos conflictos de identidad, y descriptiva, por describir las Competencias Profesionales del Nutricionista Deportivo con el fin de conocer y definir su labor a través de un proceso sistemático.

Cuanto al método elegido, la técnica Delphi, es una técnica de investigación que según ${ }^{22}$, solicita a expertos en una temática, los juicios sobre un tema en particular mediante una serie de cuestionarios cuidadosamente diseñados de forma secuencial, diseminados con información resumida y retroalimentación (feedback) de opiniones deducidas a partir de las primeras respuestas. Esta técnica esta diseñada para afrontar situaciones en las que las opiniones se imponen al conocimiento y a la especulación de los expertos, y a partir de ahí, permite sistematizar las respuestas con el objetivo de obtener el consenso de opinión más fidedigno del grupo ${ }^{23}$.
El número de sujetos para una investigación que utiliza como método la Técnica Delphi según opinan Ruiz \& Ispizua ${ }^{23}$, debe oscilar entre diez y treinta personas, "dependiendo de, si el tema se halla en una situación en la que los expertos forman una población relativamente homogénea o, más bien, los expertos se encuentren profundamente divididos entre sí y defienden posturas relativamente irreconciliables. Dado lo largo y el esfuerzo que requiere el proceso Delphi, es preferible seleccionar un panel reducido, con tal de que se asegure que están representadas en él las posturas opuestas y divergentes".

Fueron contactados en total 49 expertos de Nutrición Deportiva de cuatro países: Australia $(n=6)$, Brasil $(n=19)$, USA ( $n=15)$ y España $(n=9)$, de los cuales se logró la implicación y el compromiso de tan sólo 14 de ellos. A pesar de eso, los sujetos seleccionados (muestra) fueron suficientes para generar un conjunto de datos que permitió llegar a la información que se buscaba según la literatura. Hay que considerar, además, que la mayoría de los 14 expertos participantes poseen una destacada relevancia en el área a nivel internacional, lo que indica a priori la obtención de informaciones de estimado valor.

Los expertos participantes componen el denominado panel de expertos; son profesionales que actúan en la atención de deportistas y alumnos provenientes de centros de entrenamientos privados (clubs, gimnasios) o de centros gubernamentales (escuelas y universidades) (7), en consultas privadas (5), como docentes en universidades y centros privados (5), en empresas de consultoría orientando deportistas de alto nivel y amateurs (4) y, por último, en consulta hospitalaria (1).

Para la recopilación de los datos, se elaboró un cuestionario compuesto de preguntas abiertas (Cuadro 1) con la intención de obtener respuestas con la máxima amplitud posible, condición básica de la $1^{\text {a }}$ ronda Delphi, una ronda exploratoria que, según explica Landeta ${ }^{24}$, "cuando el estudio lo permite, se puede comenzar 
con preguntas abiertas para poder extraer ítems y cuestiones". Este guión de preguntas fue elaborado según un listado de Competencias Profesionales, identificadas tras el análisis del contenido de los siguientes artículos 2,8,25-34.

Antes que la $1^{\text {a }}$ ronda (el primer cuestionario) fuera enviada a los 14 expertos, el mismo fue sometido a un proceso de validación. Tanto los directores de la tesis como un experto en el uso de la técnica Delphi, llevaron a cabo una revisión general del primer cuestionario. Seguidamente, el mismo fue revisado por tres expertos en Nutrición originarios de tres países en los que se hablan los idiomas utilizados en la investigación: ingles, español y portugués. En este proceso se verificaron el contenido, la comprensión semántica y la coherencia lingüística de las cuestiones en cada idioma, de esta manera las sugerencias recibidas sirvieron para ajustar el instrumento.

Una vez enviada a los 14 expertos la primera ronda vía correo electrónico y habiendo recibido los cuestionarios contestados, se procedió al análisis de contenido de las respuestas. La información obtenida permitió la identificación de cuatro listados de macro categorías de Competencias Profesionales. A estos listados se añadió una escala de valoración, basada en la escala de Likert ${ }^{(3)}$, dando origen a los cuestionarios de la $2^{\mathrm{a}}$ y $3^{\mathrm{a}}$ rondas que fueron, en su determinado momento, enviados a los mismos 14 expertos. La escala aplicada era compuesta por cuatro grados de valoración (nada de acuerdo 1 punto, poco de acuerdo 2 puntos, de acuerdo 3 puntos y totalmente de acuerdo 4 puntos), y pedía la opinión o grado de acuerdo de los expertos ante cada una de las Competencias Profesionales propuestas para el Nutricionista Deportivo.

Cuanto a la validación de los cuestionarios de la $2^{\mathrm{a}}$ y $3^{\mathrm{a}}$ ronda, por haber sido elaborados a partir de los resultados de un análisis de contenido de las respuestas obtenidas en el primer cuestionario, está dotado de informaciones fiables a punto de cumplir con los requisitos de validez. Por otro lado, la escala de valoración, aplicada a los dos últimos cuestionarios, fue basada en la escala de Likert, una escala según ${ }^{36}$ es frecuentemente utilizada por proporcionar una medición o relativa capacidad de averiguar el verdadero valor de una unidad con exactitud.

Una vez obtenidos los resultados de la $2^{\text {a }}$ ronda provenientes de los 14 miembros del panel de expertos, se procedió al análisis de las respuestas a través de cálculos estadísticos descriptivos (media, moda, mediana, desviación standard). Finalmente, para la $3^{\mathrm{a}}$ y última ronda de consulta, se utilizó el mismo listado de Competencias Profesionales, salvo algunas modificaciones sugeridas por los expertos en la ronda anterior. El análisis de esta ronda se realizó de la misma manera que en la ronda anterior, esta ronda sirvió como un re-test, ya que permitió que los expertos opinasen sobre las mismas competencias después de conocer los resultados de la valoración alcanzados en la segunda ronda.

\section{RESULTADOS Y DISCUSIÓN}

\section{La mayoría de las Competencias}

Profesionales fueron bien evaluadas y presentaron un alto consenso entre los expertos. Aquellas que presentaron menor valoración y consenso fueron denominadas conflictivas y, tras una discusión donde se utilizó el juicio del investigador, el respaldo de la literatura y las opiniones de los expertos, fueron descartadas o reconsideradas. Finalmente, fueron consideradas 147 Competencias Profesionales (CP), siendo $38 \mathrm{CP}$ del saber técnico (Tabla 1), 62 del saber metodológico (Tabla 2), 24 del saber participativo (Tabla 3) y 23 del saber personal 23 (Tabla 4).

\footnotetext{
${ }^{3}$ La escala de Likert es un tipo de escala aditiva que corresponde a un nivel de medición ordinal, consistente en una serie de ítems o juicios ante los cuales se solicita la reacción del sujeto. El estímulo (ítem o sentencia) que se presenta al sujeto representa la propiedad que el investigador está interesado en medir y las respuestas son solicitadas en términos de grados de acuerdo o desacuerdo que el sujeto tenga con la sentencia en particular"35.
} 
Tabla 1. Competencias técnicas: resultados de la estadística descriptiva de los datos enviados por los expertos en Nutrición Deportiva de 4 países (Australia, Brasil, España y Estados Unidos). Datos de la segunda y tercera ronda recogidos entre julio y noviembre de 2005. En negrita se destacan los datos que presentaron alto grado de valoración entre los expertos.

\begin{tabular}{|c|c|c|c|c|c|c|c|c|}
\hline Resultados & & & רda & & & & רda & \\
\hline Competencias profesionales $\mathrm{cp}$ técnicas & $\frac{\frac{\pi}{0}}{2}$ & $\frac{\pi}{\frac{\pi}{0}}$ & $\begin{array}{l}\frac{0}{\tilde{C}} \\
\frac{.0}{0} \\
\sum\end{array}$ & 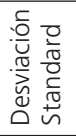 & $\frac{\frac{\pi}{0}}{\frac{0}{2}}$ & $\begin{array}{l}\frac{\pi}{0} \\
\frac{0}{2}\end{array}$ & 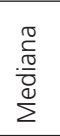 & 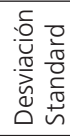 \\
\hline 1. Tener conocimientos sobre Evaluación Nutricional & 3,93 & 4,0 & 4,0 & 0,27 & 4,00 & 4,0 & 4,0 & 0,00 \\
\hline $\begin{array}{l}\text { 2. Tener conocimientos sobre Educación Alimentaria - } \\
\text { Nutricional (EAN) }\end{array}$ & 3,93 & 4,0 & 4,0 & 0,27 & 3,79 & 4,0 & 4,0 & 0,47 \\
\hline 3. Tener conocimientos sobre antropometría: & 3,79 & 4,0 & 4,0 & 0,43 & 3,79 & 4,0 & 4,0 & 0,47 \\
\hline $\begin{array}{l}\text { 3.1 Composición corporal y biotipo ideal para los diferentes } \\
\text { tipos de deporte }\end{array}$ & 3,79 & 4,0 & 4,0 & 0,43 & 3,50 & 3,0 & 3,5 & 0,67 \\
\hline 4. Tener conocimientos sobre Nutrición Clínica y saber: & 3,64 & 4,0 & 4,0 & 0,50 & 3,43 & 4,0 & 3,0 & 0,67 \\
\hline $\begin{array}{l}\text { 4.1 Detectar posibles riesgos de trastornos de la conducta } \\
\text { alimentaria }\end{array}$ & 3,36 & 3,0 & 3,0 & 0,63 & 3,64 & 4,0 & 3,5 & 0,52 \\
\hline 4.2 Interpretar pruebas analíticas - hematológicas & 3,57 & 4,0 & 4,0 & 0,65 & 3,21 & 3,0 & 3,0 & 0,54 \\
\hline $\begin{array}{l}\text { 4.3 Consecuencias de las interacciones entre fármacos y nu- } \\
\text { trientes, nutrientes y nutrientes }\end{array}$ & 3,57 & 4,0 & 4,0 & 0,51 & 3,21 & 3,0 & 3,0 & 0,54 \\
\hline $\begin{array}{l}\text { 4.4 Enfermedades de distintas naturalezas que afectan al } \\
\text { deportista: trastornos de la conducta alimentaria, } \\
\text { amenorrea, deficiencia de hierro. }\end{array}$ & 3,50 & 4,0 & 4,0 & 0,65 & 3,71 & 4,0 & 4,0 & 0,50 \\
\hline $\begin{array}{l}\text { 4.5 Identificar problemas específicos de las mujeres deportistas: } \\
\text { desórdenes alimentarios, amenorrea, osteoporosis, } \\
\text { embarazos }\end{array}$ & 3,64 & 4,0 & 4,0 & 0,50 & 3,79 & 4,0 & 4,0 & 0,47 \\
\hline $\begin{array}{l}\text { 5. Conocer los suplementos y los complementos alimentarios } \\
\text { y compuestos de ayuda ergogénica para deportistas: }\end{array}$ & 3,93 & 4,0 & 4,0 & 0,27 & 3,86 & 4,0 & 4,0 & 0,40 \\
\hline 5.1 Conocer las políticas de reglamentación & 3,64 & 4,0 & 4,0 & 0,63 & 3,50 & 3,0 & 3,0 & 0,50 \\
\hline $\begin{array}{l}\text { 5.2 Saber analizar los casos específicos en que se recomienda } \\
\text { ingerir estas sustancias }\end{array}$ & 3,86 & 4,0 & 4,0 & 0,36 & 3,79 & 4,0 & 4,0 & 0,40 \\
\hline $\begin{array}{l}\text { 6. Conocer las características pertinentes de las diversas mo- } \\
\text { dalidades deportivas (frecuencia, carga de entrenamiento, } \\
\text { periodicidad del entrenamiento en función del calendario } \\
\text { de competiciones...) }\end{array}$ & 3,64 & 4,0 & 4,0 & 0,50 & 3,71 & 4,0 & 4,0 & 0,47 \\
\hline 7. Tener conocimiento sobre la hidratación del deportista & 3,93 & 4,0 & 4,0 & 0,27 & 4,00 & 4,0 & 4,0 & 0,00 \\
\hline 8. Tener conocimiento sobre la Nutrición en general, tal como: & 3,64 & 4,0 & 4,0 & 0,63 & 3,71 & 4,0 & 4,0 & 0,50 \\
\hline 8.1 Composición de los alimentos & 3,79 & 4,0 & 4,0 & 0,58 & 3,86 & 4,0 & 4,0 & 0,40 \\
\hline 8.2 Técnicas dietéticas & 3,43 & 4,0 & 4,0 & 0,76 & 3,29 & 3,0 & 3,0 & 0,70 \\
\hline 8.3 Administración de un servicio de alimentación & 2,71 & 3,0 & 3,0 & 0,91 & 2,57 & 3,0 & 3,0 & 0,93 \\
\hline 8.4 Nutrición preventiva y otros & 3,29 & 4,0 & 3,0 & 0,73 & 3,36 & 3,0 & 3,0 & 0,65 \\
\hline $\begin{array}{l}\text { 9. Conocer métodos de investigación científica, saber revisar } \\
\text { y criticar publicaciones informativas o científicas }\end{array}$ & 3,29 & 3,0 & 3,0 & 0,73 & 3,50 & 3,0 & 3,5 & 0,52 \\
\hline $\begin{array}{l}\text { 10. Conocer métodos de recuperación de los sistemas de } \\
\text { energía }\end{array}$ & 3,79 & 4,0 & 4,0 & 0,43 & 3,86 & 4,0 & 4,0 & 0,40 \\
\hline 11. Tener conocimiento sobre los mitos alimentarios & 3,57 & 4,0 & 4,0 & 0,51 & 3,43 & 3,0 & 3,0 & 0,65 \\
\hline $\begin{array}{l}\text { 12. Tener conocimiento sobre las necesidades nutricionales } \\
\text { del deportista de acuerdo con la modalidad deportiva }\end{array}$ & 3,93 & 4,0 & 4,0 & 0,27 & 3,86 & 4,0 & 4,0 & 0,40 \\
\hline $\begin{array}{l}\text { 12.1 Conocer las demandas requeridas en función de la carga } \\
\text { (volumen }+ \text { intensidad) de la práctica deportiva }\end{array}$ & 3,86 & 4,0 & 4,0 & 0,36 & 3,93 & 4,0 & 4,0 & 0,30 \\
\hline $\begin{array}{l}\text { 12.2 Conocer como el grado de preparación física del atleta } \\
\text { puede afectar a sus necesidades nutricionales }\end{array}$ & 3,86 & 4,0 & 4,0 & 0,36 & 3,86 & 4,0 & 4,0 & 0,40 \\
\hline $\begin{array}{l}\text { 13. Saber hasta que punto los desplazamientos a } \\
\text { competiciones y entrenamientos pueden afectar el estado } \\
\text { nutricional y/o a la preparación física del deportista }\end{array}$ & 3,64 & 4,0 & 4,0 & 0,50 & 3,71 & 4,0 & 4,0 & 0,67 \\
\hline $\begin{array}{l}\text { 14. Saber como la edad y el sexo afectan la orientación alimen- } \\
\text { taria nutricional }\end{array}$ & 3,93 & 4,0 & 4,0 & 0,27 & 3,71 & 4,0 & 4,0 & 0,50 \\
\hline $\begin{array}{l}\text { 15. Conocer las diferentes técnicas y procedimientos de } \\
\text { orientación para la modificación de hábitos alimentarios }\end{array}$ & 3,79 & 4,0 & 4,0 & 0,43 & 3,71 & 4,0 & 4,0 & 0,50 \\
\hline $\begin{array}{l}\text { 16. Tener conocimiento en el área de mantenimiento, aumen- } \\
\text { to y reducción de peso }\end{array}$ & 3,93 & 4,0 & 4,0 & 0,27 & 3,93 & 4,0 & 4,0 & 0,30 \\
\hline
\end{tabular}


Tabla 1. Competencias técnicas: resultados de la estadística descriptiva de los datos enviados por los expertos en Nutrición Deportiva de 4 países (Australia, Brasil, España y Estados Unidos). Datos de la segunda y tercera ronda recogidos entre julio y noviembre de 2005. En negrita se destacan los datos que presentaron alto grado de valoración entre los expertos.

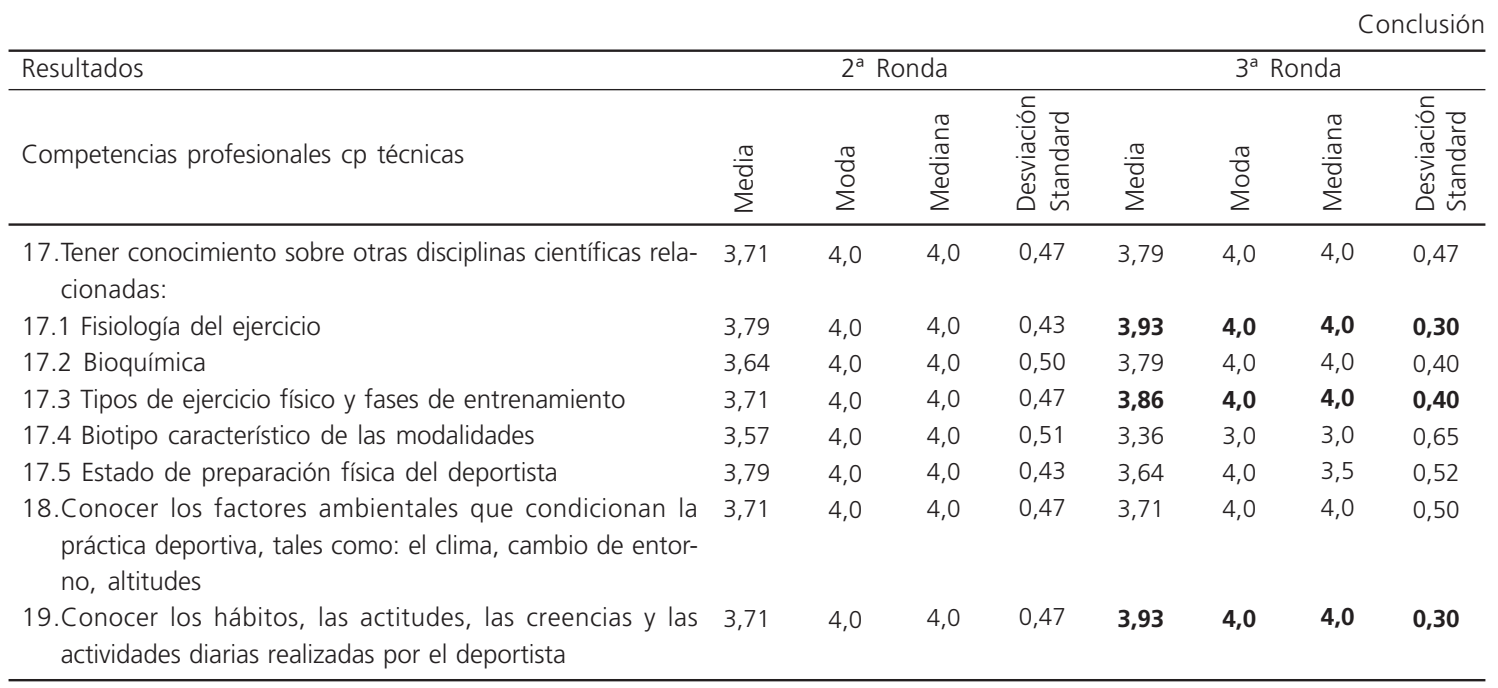

Tabla 2. Competencias metodológicas: resultados de la Estadística Descriptiva de los datos enviados por los expertos en Nutrición Deportiva de 4 países (Australia, Brasil, España y Estados Unidos). Datos de la segunda y tercera ronda recogidos entre julio y noviembre de 2005. En negrita se destacan los datos que presentaron alto grado de valoración entre los expertos.

\begin{tabular}{|c|c|c|c|c|c|c|c|c|}
\hline Resultados & & & da & & & $3^{\mathrm{a}}$ & רda & \\
\hline Competencias profesionales cp metodológicas & $\begin{array}{l}\frac{\pi}{0} \\
\sum \\
\Sigma\end{array}$ & $\begin{array}{l}\frac{\pi}{0} \\
\frac{0}{2}\end{array}$ & $\begin{array}{l}\frac{\pi}{2} \\
\frac{\pi}{0} \\
\frac{\pi}{2} \\
\sum\end{array}$ & 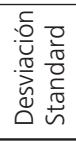 & $\frac{\frac{\pi}{0}}{\frac{d}{z}}$ & $\begin{array}{l}\frac{\pi}{0} \\
\frac{0}{2}\end{array}$ & $\begin{array}{l}\frac{\pi}{0} \\
\frac{0}{0} \\
\frac{0}{2} \\
\sum\end{array}$ & 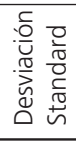 \\
\hline $\begin{array}{l}\text { 1. Saber utilizar y elegir métodos más adecuados para la } \\
\text { realización de la evaluación nutricional (anamnesis o } \\
\text { diagnóstico alimentario) }\end{array}$ & 3,93 & 4,0 & 4,0 & 0,27 & 4,00 & 4,0 & 4,0 & 0,00 \\
\hline $\begin{array}{l}\text { 2. Saber realizar educación alimentaria-nutricional a los } \\
\text { deportistas y a las personas involucradas en su alimentación, } \\
\text { considerar: }\end{array}$ & 3,86 & 4,0 & 4,0 & 0,36 & 3,93 & 4,0 & 4,0 & 0,30 \\
\hline $\begin{array}{l}\text { 2.1 El nivel de práctica deportiva (elite o practicante de ejercicio } \\
\text { físico) }\end{array}$ & 3,86 & 4,0 & 4,0 & 0,36 & 4,00 & 4,0 & 4,0 & 0,00 \\
\hline 2.2 La edad & 3,79 & 4,0 & 4,0 & 0,43 & 4,00 & 4,0 & 4,0 & 0,00 \\
\hline $2.3 \mathrm{El}$ sexo & 3,79 & 4,0 & 4,0 & 0,43 & 4,00 & 4,0 & 4,0 & 0,00 \\
\hline 2.4 Individual o colectivo & 3,79 & 4,0 & 4,0 & 0,43 & 4,00 & 4,0 & 4,0 & 0,00 \\
\hline 2.5 Modalidad deportiva & 3,79 & 4,0 & 4,0 & 0,43 & 3,93 & 4,0 & 4,0 & 0,30 \\
\hline 3. Saber realizar evaluaciones antropométricas: & 3,93 & 4,0 & 4,0 & 0,27 & 3,79 & 4,0 & 4,0 & 0,65 \\
\hline 3.1 Saber recoger e interpretar los datos antropométricos & 3,71 & 4,0 & 4,0 & 0,61 & 3,93 & 4,0 & 4,0 & 0,30 \\
\hline $\begin{array}{l}\text { 3.2 Saber realizar cálculos y determinar la composición corpo- } \\
\text { ral actual e ideal }\end{array}$ & 3,86 & 4,0 & 4,0 & 0,36 & 4,00 & 4,0 & 4,0 & 0,00 \\
\hline $\begin{array}{l}\text { 3.3 Verificar, a lo largo del tiempo, los cambios antropo-métri- } \\
\text { cos producidos }\end{array}$ & 3,93 & 4,0 & 4,0 & 0,27 & 3,93 & 4,0 & 4,0 & 0,30 \\
\hline $\begin{array}{l}\text { 4. Evaluar las necesidades nutricionales para cada deportista } \\
\text { en particular y establecer la prescripción de dietas y metas } \\
\text { de alimentación de acuerdo con: }\end{array}$ & 4,00 & 4,0 & 4,0 & 0,00 & 4,00 & 4,0 & 4,0 & 0,00 \\
\hline $\begin{array}{l}\text { 4.1 Los entrenamientos y competiciones (necesidades fisioló- } \\
\text { gicas de la modalidad deportiva) }\end{array}$ & 3,93 & 4,0 & 4,0 & 0,27 & 3,86 & 4,0 & 4,0 & 0,40 \\
\hline 4.2 La modalidad deportiva & 3,79 & 4,0 & 4,0 & 0,43 & 3,71 & 4,0 & 4,0 & 0,67 \\
\hline 4.3 Las reglas de la modalidad & 3,50 & 4,0 & 4,0 & 0,65 & 3,29 & 3,0 & 3,5 & 0,67 \\
\hline 4.4 El horario de la práctica deportiva & 3,86 & 4,0 & 4,0 & 0,36 & 3,93 & 4,0 & 4,0 & 0,30 \\
\hline 4.5 La fase de entrenamiento (fuerza, resistencia, velocidad) & 3,86 & 4,0 & 4,0 & 0,36 & 3,93 & 4,0 & 4,0 & 0,30 \\
\hline 4.6 El nivel de práctica & 3,86 & 4,0 & 4,0 & 0,36 & 3,79 & 4,0 & 4,0 & 0,47 \\
\hline 4.7 La carga (volumen + intensidad) & 3,86 & 4,0 & 4,0 & 0,36 & 3,86 & 4,0 & 4,0 & 0,40 \\
\hline
\end{tabular}


Tabla 2. Competencias metodológicas: resultados de la Estadística Descriptiva de los datos enviados por los expertos en Nutrición Deportiva de 4 países (Australia, Brasil, España y Estados Unidos). Datos de la segunda y tercera ronda recogidos entre julio y noviembre de 2005. En negrita se destacan los datos que presentaron alto grado de valoración entre los expertos.

Continuación

\begin{tabular}{|c|c|c|c|c|c|c|c|c|}
\hline Resultados & & $2^{a}$ & da & & & & רda & \\
\hline Competencias profesionales $\mathrm{cp}$ medodológicas & $\frac{\frac{\pi}{0}}{\frac{\pi}{2}}$ & $\frac{\pi}{0}$ & $\begin{array}{l}\frac{\pi}{T} \\
\frac{\pi}{0} \\
\frac{\pi}{2} \\
\sum\end{array}$ & 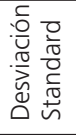 & $\frac{\frac{\pi}{0}}{2}$ & $\frac{\pi}{8}$ & $\begin{array}{l}\frac{\pi}{c} \\
\frac{\pi}{0} \\
\frac{\pi}{0} \\
\sum\end{array}$ & 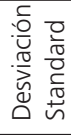 \\
\hline 4.8 La frecuencia & 3,86 & 4,0 & 4,0 & 0,36 & 3,86 & 4,0 & 4,0 & 0,40 \\
\hline 4.9 El lugar donde se practica & 3,79 & 4,0 & 4,0 & 0,43 & 3,64 & 4,0 & 4,0 & 0,52 \\
\hline 4.10 La logística & 3,57 & 4,0 & 4,0 & 0,65 & 3,36 & 4,0 & 3,5 & 0,79 \\
\hline 4.11 El sexo & 3,71 & 4,0 & 4,0 & 0,47 & 3,86 & 4,0 & 4,0 & 0,40 \\
\hline 4.12 La edad & 3,71 & 4,0 & 4,0 & 0,47 & 3,86 & 4,0 & 4,0 & 0,40 \\
\hline $\begin{array}{l}\text { 4.13 El grado de preparación física y técnica (eficacia del } \\
\text { movimiento) }\end{array}$ & 3,71 & 4,0 & 4,0 & 0,47 & 3,64 & 4,0 & 4,0 & 0,50 \\
\hline 4.14 El biotipo & 3,64 & 4,0 & 4,0 & 0,50 & 3,79 & 4,0 & 4,0 & 0,40 \\
\hline 4.15 Las preferencias personales & 3,79 & 4,0 & 4,0 & 0,43 & 3,71 & 4,0 & 4,0 & 0,50 \\
\hline 5. Verificar señales clínicas & 3,79 & 4,0 & 4,0 & 0,43 & 3,57 & 3,0 & 3,5 & 0,52 \\
\hline $\begin{array}{l}\text { 6. Saber verificar las señales clínicas de los trastornos de la } \\
\text { conducta alimentaria y como obtener ayuda de otros } \\
\text { profesionales para diagnosticarla y tratarla }\end{array}$ & 3,64 & 4,0 & 4,0 & 0,50 & 3,71 & 4,0 & 4,0 & 0,50 \\
\hline $\begin{array}{l}\text { 7. Saber prescribir suplementos y tácticas especiales para } \\
\text { deportistas (vitaminas y minerales, carbohidratos, proteínas } \\
\text { y aminoácidos, bebidas deportivas, comidas liquidas o en } \\
\text { gel, barritas deportivas, ...): }\end{array}$ & 3,86 & 4,0 & 4,0 & 0,36 & 3,79 & 4,0 & 4,0 & 0,40 \\
\hline 7.1 Saber prescribir cantidades y forma de uso & 3,86 & 4,0 & 4,0 & 0,36 & 3,79 & 4,0 & 4,0 & 0,40 \\
\hline 7.2 Saber cerciorarse de la fiabilidad del producto & 3,71 & 4,0 & 4,0 & 0,47 & 3,71 & 4,0 & 4,0 & 0,50 \\
\hline $\begin{array}{l}\text { 8. Saber interpretar los resultados de las pruebas de sangre } \\
\text { y analíticas y/o diagnosticar en conjunto con otros } \\
\text { profesionales de la salud }\end{array}$ & 3,79 & 4,0 & 4,0 & 0,58 & 3,79 & 4,0 & 4,0 & 0,47 \\
\hline $\begin{array}{l}\text { 9. Saber realizar el análisis nutricional de las dietas del } \\
\text { deportista (saber usar herramientas informáticas) }\end{array}$ & 3,93 & 4,0 & 4,0 & 0,27 & 4,00 & 4,0 & 4,0 & 0,00 \\
\hline $\begin{array}{l}\text { 10. Instruir a los deportistas a contestar los protocolos em- } \\
\text { pleados para el conocimiento de sus ingestas alimentarias }\end{array}$ & 3,79 & 4,0 & 4,0 & 0,43 & 3,79 & 4,0 & 4,0 & 0,47 \\
\hline 11. Preguntar sobre hábitos y frecuencia intestinal & 3,79 & 4,0 & 4,0 & 0,43 & 3,86 & 4,0 & 4,0 & 0,30 \\
\hline 12. Verificar el consumo de medicamentos & 3,71 & 4,0 & 4,0 & 0,47 & 3,64 & 4,0 & 4,0 & 0,50 \\
\hline $\begin{array}{l}\text { 13. Saber educar sobre hábitos y estilos de vida saludables } \\
\text { (cuestiones higiénicas y sanitarias) }\end{array}$ & 3,50 & 4,0 & 4,0 & 0,65 & 3,36 & 4,0 & 4,0 & 0,81 \\
\hline $\begin{array}{l}\text { 14. Evaluar la implicación del deportista con lo prescrito y a lo } \\
\text { orientado anteriormente, y las razones de éxitos o fracasos }\end{array}$ & 3,86 & 4,0 & 4,0 & 0,36 & 3,71 & 4,0 & 4,0 & 0,50 \\
\hline $\begin{array}{l}\text { 15. Identificar los problemas que dificultan la adherencia a la } \\
\text { pauta propuesta e incidir sobre ella }\end{array}$ & 3,86 & 4,0 & 4,0 & 0,36 & 3,71 & 4,0 & 4,0 & 0,50 \\
\hline $\begin{array}{l}\text { 16. Orientar sobre los mitos alimentarios existentes y los temas } \\
\text { típicos de cada modalidad }\end{array}$ & 3,71 & 4,0 & 4,0 & 0,47 & 3,64 & 4,0 & 3,5 & 0,52 \\
\hline 17. Evaluar la efectividad del tratamiento dietético prescrito & 3,86 & 4,0 & 4,0 & 0,36 & 3,86 & 4,0 & 4,0 & 0,40 \\
\hline $\begin{array}{l}\text { 18. Planificar estrategias alimentarias cuantificadas y específi- } \\
\text { cas a cada deportista, llevando en consideración la rutina } \\
\text { o calendario de actividades y de competición de los } \\
\text { deportistas: }\end{array}$ & 3,93 & 4,0 & 4,0 & 0,27 & 3,93 & 4,0 & 4,0 & 0,30 \\
\hline $\begin{array}{l}\text { 18.1 Saber orientar para las comidas o tentempiés más } \\
\text { adecuados para antes, durante y después del ejercicio } \\
\text { físico o competición }\end{array}$ & 3,93 & 4,0 & 4,0 & 0,27 & 4,00 & 4,0 & 4,0 & 0,00 \\
\hline $\begin{array}{l}\text { 18.2 Saber orientar para las comidas realizadas dentro o fuera } \\
\text { de la institución o centro de entrenamiento }\end{array}$ & 3,93 & 4,0 & 4,0 & 0,27 & 4,00 & 4,0 & 4,0 & 0,00 \\
\hline 18.3 Considerar los cambios del estado emocional & 3,71 & 4,0 & 4,0 & 0,47 & 3,64 & 4,0 & 4,0 & 0,50 \\
\hline 18.4 Considerar cambios de la preparación física & 3,79 & 4,0 & 4,0 & 0,43 & 3,86 & 4,0 & 4,0 & 0,40 \\
\hline $\begin{array}{l}\text { 19. Conocer las necesidades especiales de los deportistas y } \\
\text { proporcionar una orientación dietética dirigida tanto a sus } \\
\text { objetivos deportivos como a los de salud: }\end{array}$ & 3,79 & 4,0 & 4,0 & 0,43 & 3,86 & 4,0 & 4,0 & 0,40 \\
\hline
\end{tabular}


Tabla 2. Competencias metodológicas: resultados de la Estadística Descriptiva de los datos enviados por los expertos en Nutrición Deportiva de 4 países (Australia, Brasil, España y Estados Unidos). Datos de la segunda y tercera ronda recogidos entre julio y noviembre de 2005. En negrita se destacan los datos que presentaron alto grado de valoración entre los expertos.

Conclusión

\begin{tabular}{|c|c|c|c|c|c|c|c|c|}
\hline Resultados & & & & & & $3^{a}$ & & \\
\hline Competencias profesionales $\mathrm{cp}$ metodológicas & $\begin{array}{l}\frac{\pi}{0} \\
\frac{\pi}{2}\end{array}$ & $\begin{array}{l}\frac{\pi}{0} \\
\frac{0}{2}\end{array}$ & $\begin{array}{l}\frac{\pi}{\sigma} \\
\frac{\pi}{0} \\
\frac{\pi}{0} \\
\sum\end{array}$ & 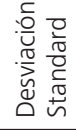 & $\begin{array}{l}\frac{\pi}{0} \\
\frac{\pi}{2}\end{array}$ & $\begin{array}{l}\frac{\pi}{0} \\
\frac{0}{2}\end{array}$ & $\begin{array}{l}\frac{\pi}{\frac{\pi}{0}} \\
\frac{\pi}{0} \\
\frac{\pi}{2}\end{array}$ & 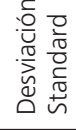 \\
\hline $\begin{array}{l}\text { 19.1 Verificar cambios en los resultados del rendimiento físico } \\
\text { y deportivo después del inicio de la intervención }\end{array}$ & 3,79 & 4,0 & 4,0 & 0,43 & 3,86 & 4,0 & 4,0 & 0,40 \\
\hline $\begin{array}{l}\text { 20. Evaluar la disminución y la desaparición de las molestias } \\
\text { planteadas en la primera consulta }\end{array}$ & 3,71 & 4,0 & 4,0 & 0,47 & 3,86 & 4,0 & 4,0 & 0,40 \\
\hline $\begin{array}{l}\text { 21. Conseguir una óptima recuperación de las reservas } \\
\text { energéticas utilizadas en los entrenamientos y } \\
\text { competiciones a través de la orientación alimentario - } \\
\text { nutricional }\end{array}$ & 3,93 & 4,0 & 4,0 & 0,27 & 4,00 & 4,0 & 4,0 & 0,00 \\
\hline 22. Determinar y educar sobre estrategias de hidratación & 3,93 & 4,0 & 4,0 & 0,27 & 4,00 & 4,0 & 4,0 & 0,00 \\
\hline $\begin{array}{l}\text { 23. Realizar talleres sobre la alimentación en la actividad física } \\
\text { a los deportistas y a todos los responsables de la } \\
\text { alimentación de los deportistas }\end{array}$ & 3,50 & 4,0 & 4,0 & 0,65 & 3,50 & 3,0 & 3,5 & 0,67 \\
\hline $\begin{array}{l}\text { 24. Realizar monitorización de los aspectos bioquímicos y } \\
\text { fisiológicos en conjunto con el equipo medico: }\end{array}$ & 3,86 & 4,0 & 4,0 & 0,36 & 3,79 & 4,0 & 4,0 & 0,40 \\
\hline 24.1 Saber trabajar con un equipo multidisciplinar & 3,86 & 4,0 & 4,0 & 0,36 & 3,86 & 4,0 & 4,0 & 0,40 \\
\hline $\begin{array}{l}\text { 25. Realizar grupos de discusión con los deportistas y } \\
\text { entrenadores }\end{array}$ & 3,71 & 4,0 & 4,0 & 0,47 & 3,64 & 4,0 & 4,0 & 0,69 \\
\hline 26. Realizar actividades educativas en grupo & 3,57 & 4,0 & 4,0 & 0,65 & 3,64 & 4,0 & 4,0 & 0,52 \\
\hline $\begin{array}{l}27 \text { Verificar qué estrategias funcionan mejor individualmente } \\
\text { o en grupo }\end{array}$ & 3,79 & 4,0 & 4,0 & 0,43 & 3,64 & 4,0 & 4,0 & 0,52 \\
\hline 28. Impartir clases de técnica culinaria & 3,00 & 4,0 & 3,0 & 0,96 & 2,50 & 2,0 & 2,0 & 0,52 \\
\hline 29. Realizar visitas guiadas al supermercado & 2,93 & 3,0 & 3,0 & 0,83 & 2,57 & 2,0 & 2,0 & 0,47 \\
\hline $\begin{array}{l}\text { 30. Saber tratar con deportistas que compiten en modalida- } \\
\text { des deportivas reguladas por el peso de los participantes }\end{array}$ & 3,79 & 4,0 & 4,0 & 0,43 & 3,86 & 4,0 & 4,0 & 0,40 \\
\hline 31.Saber seleccionar y/o desarrollar materiales educativos & 3,64 & 4,0 & 4,0 & 0,50 & 3,43 & 4,0 & 3,5 & 0,79 \\
\hline $\begin{array}{l}\text { 32. Desarrollar contenidos de cursos, ponencias, charlas y } \\
\text { talleres }\end{array}$ & 3,64 & 4,0 & 4,0 & 0,63 & 3,57 & 3,0 & 3,5 & 0,52 \\
\hline $\begin{array}{l}\text { 33. Conocimientos básicos para redacción de artículos cientí- } \\
\text { ficos y/o divulgativos para publicación }\end{array}$ & 3,29 & 4,0 & 4,0 & 0,83 & 3,14 & 3,0 & 3,0 & 0,83 \\
\hline 34.Exponer materiales informativos en lugares estratégicos & 3,43 & 4,0 & 4,0 & 0,65 & 3,14 & 3,0 & 3,0 & 0,83 \\
\hline $\begin{array}{l}\text { 35. Saber orientar en los casos específicos cuando el deportista } \\
\text { posee un presupuesto limitado o falta de patrocinadores }\end{array}$ & 3,79 & 4,0 & 4,0 & 0,43 & 3,71 & 4,0 & 4,0 & 0,67 \\
\hline $\begin{array}{l}\text { 36. Dar asistencia domiciliaria para observar: las prácticas de } \\
\text { compra de los géneros alimenticios, la preparación de los } \\
\text { alimentos, entre otras. }\end{array}$ & 2,93 & 3,0 & 3,0 & 0,83 & 2,64 & 3,0 & 3,0 & 0,93 \\
\hline
\end{tabular}

En las Tablas 1, 2, 3, y 4 quedan reflejadas todas las competencias identificadas en la $1^{\text {a }}$ ronda del Estudio Delphi, bien como los resultados obtenidos tras la aplicación de la escala de valoración en la $2^{\mathrm{a}}$ y $3^{\mathrm{a}}$ ronda. Los resultados fueron vistos de dos ópticas distintas: primera según el consenso entre los expertos (el grado de acuerdo) representados por los valores numéricos de la Desviación Standard (DS) (DS=0 - consenso absoluto; DS $>0<0.5$ - consenso suficiente; $D S>0.50 y<0.96$ - menor consenso) y segundo por el grado de valoración representados por los demás resultados de la estadística descriptiva: la media (M) $(M<3.5$ - menor valoración; $M>3.86$ mayor valoración), la moda (valores 2 y 1 - menor valoración) y mediana (valores $<3$ - menor valoración).

Se notó una ligera diferencia en el grado de valoración o puntuación que recibieron las competencias según la nacionalidad de los sujetos. Solamente se puede hablar de tendencias, ya que el número de expertos participantes en esta 
Tabla 3. Competencias participativas: resultados de la Estadística Descriptiva de los datos enviados por los expertos en Nutrición Deportiva de 4 países (Australia, Brasil, España y Estados Unidos). Datos de la segunda y tercera ronda recogidos entre julio y noviembre de 2005. En negrita se destacan los datos que presentaron alto grado de valoración entre los expertos.

\begin{tabular}{|c|c|c|c|c|c|c|c|c|}
\hline Resultados & & $2^{\mathrm{a}}$ & & & & & רda & \\
\hline Competencias profesionales $\mathrm{cp}$ participativas & $\frac{\frac{\pi}{0}}{\frac{0}{2}}$ & $\frac{\sqrt{0}}{0}$ & $\begin{array}{l}\frac{\pi}{c} \\
\frac{\pi}{0} \\
\frac{\pi}{2} \\
\sum\end{array}$ & 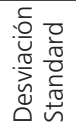 & $\frac{\pi}{\frac{\pi}{0}}$ & $\begin{array}{l}\frac{\pi}{0} \\
\frac{0}{2}\end{array}$ & 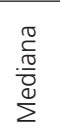 & 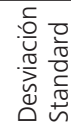 \\
\hline $\begin{array}{l}\text { 1. Comprender el motivo de la consulta del deportista para } \\
\text { una orientación alimentario - nutricional }\end{array}$ & 3,86 & 4,0 & 4,0 & 0,36 & 3,93 & 4,0 & 4,0 & 0,00 \\
\hline $\begin{array}{l}\text { 2. Contactarse con técnicos y responsables de la alimentación } \\
\text { del deportista para la obtención de informaciones } \\
\text { complementarias sobre cual es su alimentación }\end{array}$ & 3,50 & 4,0 & 4,0 & 0,65 & 3,50 & 4,0 & 4,0 & 0,67 \\
\hline $\begin{array}{l}\text { 3. Obtener información sobre los entrenamientos y las } \\
\text { competiciones a través del equipo técnico: preparador } \\
\text { físico, técnico, entrenador y los propios deportistas. }\end{array}$ & 3,86 & 4,0 & 4,0 & 0,36 & 3,86 & 4,0 & 4,0 & 0,39 \\
\hline 4. Saber trabajar con un equipo multidisciplinar & 3,93 & 4,0 & 4,0 & 0,27 & 3,86 & 4,0 & 4,0 & 0,39 \\
\hline 5. Participar en estudios de colaboración y grupos de estudios & 3,21 & 3,0 & 3,0 & 0,70 & 3,43 & 4,0 & 4,0 & 0,67 \\
\hline $\begin{array}{l}\text { 6. Actualizarse a través de publicaciones de la literatura cien- } \\
\text { tífica reciente, páginas Web oficiales del deporte, simposios, } \\
\text { congresos y seminarios (formación continuada) }\end{array}$ & 3,93 & 4,0 & 4,0 & 0,27 & 3,93 & 4,0 & 4,0 & 0,29 \\
\hline $\begin{array}{l}\text { 7. Saber derivar al deportista a otro profesional cuando sea } \\
\text { necesario }\end{array}$ & 4,00 & 4,0 & 4,0 & 0,00 & 3,86 & 4,0 & 4,0 & 0,39 \\
\hline $\begin{array}{l}\text { 8. Apoyar y animar al deportista a que siga de forma estricta } \\
\text { las recomendaciones recibidas }\end{array}$ & 3,86 & 4,0 & 4,0 & 0,36 & 3,79 & 4,0 & 4,0 & 0,45 \\
\hline $\begin{array}{l}\text { 9. Educar a los deportistas sobre los beneficios de estrategias } \\
\text { alimentarias especificas y su capacidad de mejorar el } \\
\text { rendimiento deportivo }\end{array}$ & 3,86 & 4,0 & 4,0 & 0,36 & 3,86 & 4,0 & 4,0 & 0,39 \\
\hline $\begin{array}{l}\text { 10. Obtener el apoyo de los técnicos y preparadores físicos del } \\
\text { equipo }\end{array}$ & 3,79 & 4,0 & 4,0 & 0,43 & 3,64 & 4,0 & 4,0 & 0,51 \\
\hline $\begin{array}{l}\text { 11. Familiarizarse con la terminología utilizada en las diversas } \\
\text { modalidades deportivas }\end{array}$ & 3,79 & 4,0 & 4,0 & 0,43 & 3,64 & 4,0 & 4,0 & 0,51 \\
\hline 12. Identificar dificultades y dudas más frecuentes & 3,86 & 4,0 & 4,0 & 0,36 & 3,79 & 4,0 & 4,0 & 0,45 \\
\hline $\begin{array}{l}\text { 13. Realizar la consulta en el lugar donde entrenan los } \\
\text { deportistas }\end{array}$ & 2,79 & 2,0 & 3,0 & 0,80 & 2,86 & 3,0 & 3,0 & 0,94 \\
\hline $\begin{array}{l}\text { 14. Verificar la valoración del deportista hacia la alimentación y } \\
\text { su motivación para realizar cambios }\end{array}$ & 3,71 & 4,0 & 4,0 & 0,47 & 3,71 & 4,0 & 4,0 & 0,49 \\
\hline $\begin{array}{l}\text { 15. Transmitir las informaciones de manera breve, concreta y } \\
\text { aplicable }\end{array}$ & 3,79 & 4,0 & 4,0 & 0,43 & 3,93 & 4,0 & 4,0 & 0,29 \\
\hline 16. Considerar la cultura, las actitudes y creencias del deportista & 3,79 & 4,0 & 4,0 & 0,43 & 3,71 & 4,0 & 4,0 & 0,49 \\
\hline $\begin{array}{l}\text { 17. Ir personalmente a los entrenamientos para entender mejor } \\
\text { las características de las modalidades deportivas nunca } \\
\text { antes trabajadas por el profesional }\end{array}$ & 3,43 & 4,0 & 4,0 & 0,76 & 3,36 & 3,0 & 3,0 & 0,65 \\
\hline $\begin{array}{l}\text { 18. Motivar al deportista para no abandonar el tratamiento y } \\
\text { buscar otras fórmulas más rápidas }\end{array}$ & 3,57 & 4,0 & 4,0 & 0,65 & 3,79 & 4,0 & 4,0 & 0,45 \\
\hline $\begin{array}{l}\text { 19. Mantenerse informado sobre las estrategias alimentarias } \\
\text { de suplementación }\end{array}$ & 3,79 & 4,0 & 4,0 & 0,43 & 3,93 & 4,0 & 4,0 & 0,29 \\
\hline 20. Mantener conducta ética y reputación profesional & 4,00 & 4,0 & 4,0 & 0,00 & 3,93 & 4,0 & 4,0 & 0,29 \\
\hline $\begin{array}{l}\text { 21. Saber tratar con profesionales que no son del área de la } \\
\text { Nutrición Deportiva y que se consideran expertos en esa } \\
\text { área }\end{array}$ & 3,86 & 4,0 & 4,0 & 0,36 & 3,71 & 4,0 & 4,0 & 0,45 \\
\hline 22. Saber tratar con vendedores de productos dietéticos & 3,64 & 4,0 & 4,0 & 0,74 & 3,57 & 4,0 & 4,0 & 0,67 \\
\hline 23. Saber tratar, tanto individualmente como en equipo & 3,86 & 4,0 & 4,0 & 0,36 & 3,93 & 4,0 & 4,0 & 0,29 \\
\hline $\begin{array}{l}\text { 24. Aceptar consejos que los profesionales más experientes } \\
\text { pueden ofrecer }\end{array}$ & 3,93 & 4,0 & 4,0 & 0,27 & 3,86 & 4,0 & 4,0 & 0,39 \\
\hline 25.Saber vender la experiencia y el conocimiento profesional & 3,71 & 4,0 & 4,0 & 0,47 & 3,86 & 4,0 & 4,0 & 0,39 \\
\hline
\end{tabular}


investigación no permite realizar pruebas de significación estadística. Así, los países de habla inglesa, estos son Australia y Estados Unidos, proporcionaron una valoración ligeramente más positiva para todas las clases de competencias si comparados a los países de idiomas latinos, a saber, Brasil y España. Entre los países de habla latina, parece ser que los expertos brasileños valoraron más las CP Metodológicas y Participativas con respecto a los españoles, quienes valoraron más las CP Técnicas y Personales.

En general las categorías de competencias participativas y personales presentaron un mayor consenso y valoración, si comparadas a las categorías técnicas y metodológicas. En la $3^{\mathrm{a}}$ ronda la valoración de las 4 clases de competencias demostró una ligera disminución del consenso, a pesar de que los valores son pocos significativos, y todas aquellas competencias menos valoradas fueron también motivo de menor consenso.

Era de esperarse de que algunas competencias no fueran bien valorizadas o no presentaran un alto grado de consenso. Pero, afortunadamente, la mayoría de ellas fueron bien evaluadas y presentaron un alto consenso entre los expertos. Aquellas que presentaron menor

Tabla 4. Competencias personales: resultados de la Estadística Descriptiva de los datos enviados por los expertos en Nutrición Deportiva de 4 países (Australia, Brasil, España y Estados Unidos). Datos de la segunda y tercera ronda recogidos entre julio y noviembre de 2005. En negrita se destacan los datos que presentaron alto grado de valoración entre los expertos.

\begin{tabular}{|c|c|c|c|c|c|c|c|c|}
\hline Resultados & & $2^{a}$ & da & & & $3^{a}$ & da & \\
\hline Competencias profesionales cp personales & $\frac{.0}{\frac{0}{0}}$ & $\begin{array}{l}\frac{0}{0} \\
\frac{0}{\Sigma}\end{array}$ & 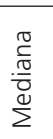 & 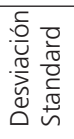 & $\frac{\frac{\pi}{0}}{\frac{d}{2}}$ & $\begin{array}{l}\frac{0}{0} \\
\frac{0}{\Sigma}\end{array}$ & 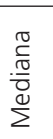 & 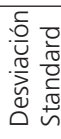 \\
\hline $\begin{array}{l}\text { 1. Disfrutar de esta profesión así como de sus ámbitos de } \\
\text { actuación }\end{array}$ & 3,71 & 4,0 & 4,0 & 0,47 & 3,64 & 4,0 & 4,0 & 0,50 \\
\hline 2. Creer en esta área de actuación de la nutrición & 4,00 & 4,0 & 4,0 & 0,00 & 3,86 & 4,0 & 4,0 & 0,36 \\
\hline $\begin{array}{l}\text { 3. Buscar las novedades e intentar actualizarse constante- } \\
\text { mente }\end{array}$ & 4,00 & 4,0 & 4,0 & 0,00 & 3,86 & 4,0 & 4,0 & 0,36 \\
\hline 4. Ser dinámico & 3,79 & 4,0 & 4,0 & 0,58 & 3,71 & 4,0 & 4,0 & 0,47 \\
\hline 5. Ser curioso y estudioso & 3,93 & 4,0 & 4,0 & 0,27 & 3,86 & 4,0 & 4,0 & 0,36 \\
\hline 6. Ser dedicado y disciplinado & 3,79 & 4,0 & 4,0 & 0,58 & 3,86 & 4,0 & 4,0 & 0,36 \\
\hline 7. Ser innovador & 3,86 & 4,0 & 4,0 & 0,53 & 3,79 & 4,0 & 4,0 & 0,43 \\
\hline 8. Ser comunicativo & 4,00 & 4,0 & 4,0 & 0,00 & 3,93 & 4,0 & 4,0 & 0,27 \\
\hline 9. Ser creativo & 3,71 & 4,0 & 4,0 & 0,61 & 3,71 & 4,0 & 4,0 & 0,47 \\
\hline 10. Ser decisivo & 3,86 & 4,0 & 4,0 & 0,36 & 3,71 & 4,0 & 4,0 & 0,47 \\
\hline 11. Ser práctico & 4,00 & 4,0 & 4,0 & 0,00 & 3,93 & 4,0 & 4,0 & 0,27 \\
\hline 12. Ser perseverante y paciente & 3,86 & 4,0 & 4,0 & 0,36 & 3,86 & 4,0 & 4,0 & 0,36 \\
\hline 13. Ser independiente & 3,43 & 4,0 & 4,0 & 0,76 & 3,36 & 4,0 & 4,0 & 0,93 \\
\hline 14. Ser flexible & 3,86 & 4,0 & 4,0 & 0,36 & 3,79 & 4,0 & 4,0 & 0,43 \\
\hline 15. Ser activo y practicar lo que se enseña & 3,64 & 4,0 & 4,0 & 0,84 & 3,79 & 4,0 & 4,0 & 0,43 \\
\hline 16. Saber aconsejar & 3,86 & 4,0 & 4,0 & 0,36 & 3,86 & 4,0 & 4,0 & 0,36 \\
\hline 17. Saber relacionarse & 3,93 & 4,0 & 4,0 & 0,27 & 3,93 & 4,0 & 4,0 & 0,27 \\
\hline 18. Saber negociar & 3,79 & 4,0 & 4,0 & 0,43 & 3,71 & 4,0 & 4,0 & 0,47 \\
\hline 19. Saber distribuir bien el tiempo & 3,71 & 4,0 & 4,0 & 0,47 & 3,71 & 4,0 & 4,0 & 0,47 \\
\hline 20. Estar motivado & 3,86 & 4,0 & 4,0 & 0,36 & 3,86 & 4,0 & 4,0 & 0,36 \\
\hline 21. Demostrar empatía & 3,79 & 4,0 & 4,0 & 0,43 & 3,86 & 4,0 & 4,0 & 0,36 \\
\hline $\begin{array}{l}\text { 22. Haber practicado o practicar algún deporte o actividad } \\
\text { física }\end{array}$ & 3,29 & 4,0 & 3,0 & 0,83 & 3,43 & 4,0 & 3,0 & 0,65 \\
\hline 23. Tener buen humor y actitud & 3,71 & 4,0 & 4,0 & 0,47 & 3,71 & 4,0 & 4,0 & 0,47 \\
\hline 24. Tener carácter & 3,57 & 4,0 & 4,0 & 0,85 & 3,64 & 4,0 & 4,0 & 0,63 \\
\hline 25. Tener capacidad de convicción y liderazgo & 3,50 & 4,0 & 4,0 & 0,94 & 3,71 & 4,0 & 4,0 & 0,47 \\
\hline
\end{tabular}


Cuadro 1. Cuestionario de la $1^{\text {a }}$ ronda del estudio Delphi

\section{$1^{\text {a }}$ Parte: Conociendo al experto}

Por favor responda en el espacio en blanco las siguientes preguntas personales con la amplitud que considere conveniente:

1) Describa su formación académica (general y específica en Nutrición Deportiva).

2) ¿Qué lo llevo a trabajar en el ámbito de la Nutrición Deportiva?

3) ¿Cuál fue su primer trabajo como Nutricionista Deportivo?

4) ¿Dónde trabaja actualmente?

5) ¿Tuvo alguna otra experiencia laboral, fuera de la Nutrición Deportiva, que pudo haber contribuido para que hoy esté trabajando en esta área?

6) ¿Hasta que punto cree que esta experiencia laboral previa fue importante para que hoy esté trabajando en Nutrición Deportiva?

7) ¿Posee actualmente un contrato laboral como Nutricionista Deportivo? Si es así, describa cuales son sus responsabilidades de acuerdo con este contrato.

\section{$2^{\text {a }}$ Parte: Conociendo la labor del Nutricionista Deportivo}

Por favor responda en el espacio en blanco las siguientes preguntas según su experiencia profesional como Nutricionista Deportivo con la amplitud que considere conveniente:

8) ¿Con qué tipo de deportista o atleta suele tratar? (edad, sexo, deporte o actividad física, nivel de entrenamiento).

9) ¿Hasta qué punto cree usted que sus funciones son distintas cuando trata con un atleta o equipo de deportistas amateur, o bien cuando trata con un atleta o equipo de deportistas profesionales?

10) ¿Cuáles cree usted que son los principales objetivos en una primera consulta realizada a un deportista individualmente?

11) ¿Cuáles cree usted que son los principales objetivos en las siguientes consultas?

12) ¿Cuáles cree usted que son los principales objetivos de una primera consulta realizada a un grupo de deportistas?

13) ¿Cuáles cree usted que son los principales objetivos en las siguientes consultas?

14) En relación a las preguntas 11, 12, 13 y 14, ¿cree usted que estos objetivos de las consultas cambian significativamente cuando se trata de deportistas de diferentes modalidades deportivas? Y si es así, ¿porqué, y en qué sentido?

15) Si tuviera que trabajar con un deporte con el que nunca antes había trabajado, ¿buscaría algún tipo de información adicional? Y en este caso, ¿donde buscaría esta información?

16) ¿Ha tratado profesionalmente con deportistas que padezcan problemas alimentarios? Si es así, ¿que tipo de problema alimentario ve usted con más frecuencia? En estos casos, ¿cómo intenta solucionarlos?

17) ¿Utiliza alguna táctica alimenticia especial con sus clientes (suplementos nutricionales, comidas ya preparadas, alimentos diseñados especialmente para deportistas)? Especifique.

18) ¿Cree usted que la función del Nutricionista Deportivo es la prescripción de suplementos ergogénicos de distinta naturaleza?

19) ¿Trabaja usted junto a un equipo multidisciplinar? ¿Cree usted en la importancia de trabajar en equipo? ¿Por qué?

20) ¿De qué manera sus clientes suelen recibir y aceptar sus orientaciones?

21) ¿Cuáles cree usted que son los mayores desafíos a los que se enfrenta profesionalmente?

22) ¿Realiza usted habitualmente algún tipo de Formación Continuada?, ¿de qué tipo?

23) ¿Que consejos daría usted para cualquier nutricionista que quisiera trabajar o iniciarse en la nutrición deportiva?

24) ¿Cuál cree usted que es el perfil personal, característico de un profesional de la Nutrición Deportiva?

valoración y consenso fueron denominadas CP conflictivas y, tras una discusión donde se utilizó el juicio del investigador, el respaldo de la literatura y las opiniones de los expertos, fueron descartadas o reconsideradas. Al principio habían $11 \mathrm{CP}$ conflictivas (28\%) de la categoría de competencias técnicas, aunque después de una discusión se pudieron reconsiderar todas. En la categoría metodológica habían 20 CP conflictivas (29\%) de las cuales fueron reconsideradas 15 . En la categoría participativa habían 5 CP conflictivas
(23\%) de las cuales 3 fueron reconsideradas $y_{1}$ por último, la categoría personal presentó 3 competencias conflictivas (13\%), pudiendo ser reconsiderada apenas 1 de ellas tras la discusión ${ }^{(4)}$.

Las categorías de CP que obtuvieron el más alto nivel de valoración y de consenso fueron las Participativas y Personales (el saber, estar y ser). Estas clases de CP son de naturaleza muy distinta de las competencias Técnicas y Metodológicas, pues están formadas por componentes subjetivos como son la personalidad, la percepción y el

\footnotetext{
4 Porcentaje estimado.
} 
sentido crítico, algo que últimamente se viene valorando mucho en cualquier área o ámbito profesional.

Por esta razón creemos que el modelo de las Competencias profesionales incita a la revisión de los objetivos educativos, de las concepciones pedagógicas y de los contenidos abordados durante la formación, permitiendo elevar la calidad de la educación superior en Nutrición y Dietética y ampliar las posibilidades de trabajo para los profesionales ${ }^{37-39}$.

Se espera, sobretodo que estas informaciones sean de gran utilidad para las instituciones académicas que imparten la carrera de Nutrición y Dietética Humana a la hora de formular qué contenidos deben ser incluidos en la disciplina de Nutrición Deportiva, la cual debería formar parte de los planes curriculares de esta carrera. Dicha disciplina, pudiendo ser denominada Nutrición Deportiva ${ }^{(5)}$, podrá abordar, con los resultados aquí presentados, temas como funciones, habilidades, calidades y competencias que deben poseer los profesionales que trabajan en el mundo de la Nutrición Deportiva, en especial los Nutricionistas Deportivos.

\section{COLABORADORES}

M. L. BELLOTTO realizou a revisão da literatura, a formulação do objetivo de estudo, a definição da estrutura metodológica e sua aplicação para a investigação, a análise dos dados, a elaboração da discussão e a conclusão do estudo. I. P. LINARES revisou e orientou a elaboração do presente artigo. Revisou os itens requisitos indispensáveis para a publicação de artigos originais.

\section{REFERENCIAS}

1. American Dietetic Association. Dietetics Education Task Force Report and Recommendations. 2005 [aceso en 2005 Jun 26]. Disponible en: <http:// www.cdrnet.org/PDFs/hod_sm_21_dietedtask forcerep.pdf>.
2. Baldwin CJ. Food and fitness careers for dietitians. J Am Diet Assoc. 2002; 102(11):1611-2.

3. Rosebloom CA. Sports Nutrition: a guide for the professional working with active people. 3a. ed. Illinois: American Dietetic Association; 1993.

4. Clark N. Identifying the educational needs of aspiring sports nutritionists. J Am Diet Assoc. 2000; 10(12):1522-4.

5. Konopka P. La alimentación del deportista: deportes técnicas. Martinez Roca; 1988.

6. Brouns F. Necesidades nutricionales de los atletas. Barcelona: Editorial Paidotribo; 1995.

7. Wolinsky I. Nutrition in exercise and sport. 3rd ed. Flórida: CRC Pres; 1997.

8. Shattuck D. Sports nutritionists fuel the competitive edge. J Am Diet Assoc. 2001; 101(5):517-8.

9. Hirschbruch MD, Carcalho JR. Nutrição esportiva: uma visão prática. São Paulo: Manole; 2002.

10. Bellotto ML. La opinión de los alumnos respecto a un curso de nutrición: el caso de la Unimep - Brasil. Póster presentado en el II Congreso de la Associação Espanhola de Dietética Nutricionista; 2004 Oct. España.

11. Meyer N. Summary of surveys from National Organizations for Sports Dietitians and countries interested in Sports Nutrition. Compiled at American College of Sports Medicine and a report from the international Sports Nutrition Safári. Held in South África; 2005 Sep; África.

12. Le Boterf G. Ingeniería de las competencias. Barcelona: Gestión; 2000/2001.

13. Bellotto ML. Las competencias profesionales del nutricionista deportivo [tesis]. Lleida: Universitad de Lleida; 2006.

14. Levy-Leboyer C. Gestión de las competencias. Barcelona: Gestión; 1997/2000.

15. Bunk GP. "La transformación de las competencias en la formación y perfeccionamiento profesionales de la R.F.A." Berlin CEDEFOP. Rev Eur Form Prof. 1994; 2(1):8-14.

16. Touger-Decker R. Preparing dietetic professional for practice in the 21st Century: How can educational programs respond to changes in health care? J Am Diet Assoc. 1998; 14(6):535-9.

17. O'Sullivan-Maillet J. The competencies continuum in nutrition. Nutrition. 1997; 13(9):840-1.

18. Nijhof WJ, Streumer JN. Key qualifications in work and education. Netherlands: Kluwer Academic Publishers; 1998.

\footnotetext{
$\overline{5}$ O recibir otras denominaciones y títulos, como por ejemplo Nutrición Aplicada a la Actividad Física y al Deporte, o cualquier otro nombre que la comunidad científica pueda estar de acuerdo.
} 
19. Dahl L, Leonberg B. JCAHO and CDR: Meeting the competence challenge. J Am Diet Assoc. 1998; 98(5):589-90.

20. Fuhrman M P. Issues facing dietetics professionals: challenges and opportunities. J Am Diet Assoc. 2002; 102(11):1618-20.

21. Ruiz J। O. Metodología de la investigación cualitativa. 2a. ed. Bilbao: Universidad de Deusto; 1999.

22. Delbeca $A$ L, Van de Ven $A H$, Gustafson $D H$. Técnicas grupales para la planeación. México: Editorial Trillas; 1984.

23. Ruiz J। O. Ispizua, M A. La descodificación de la vida cotidiana. Bilbao: Universidad de Deusto; 1994.

24. Landeta J. El método Delphi: Una técnica de previsión del futuro. Barcelona: Editorial Arien; 2002.

25. Best JW. Como investigar en educación. 3a. ed. Madrid: Edciones Morata; 1974.

26. Burke LM. Developing a sports nutrition website: an interview with Michelle Minehen. Int J S Nutr Exerc Metab. 2001a; 11(3):513-5.

27. Burke LM. Practical sport nutrition: an interview with Linda Houtkooper. Int J Sport Nutr Exerc Metab. 2001b; 11(2):264-7.

28. Burke LM. Feeding ultra - endurance athletes: an interview with Dr. Helen O'Connor and Gregory Cox. Int J Sport Nutr Exerc Metab. 2002; 12(4): 490-4.

29. Burke LM. An interview with Nelson Steen. Int J Sport Nutr Exerc Metab. 2003a; 13(1):117-21.

30. Burke LM. Nutrition for open water sailing: an interview with Jeni Pearce, sport dietitian. Int J Sport Nutr Exerc Metab. 2003b; 13(2):244-9.
31. Clark K. Roundtable: consulting in sport nutrition. Int J Sport Nutr. 1996; 6(2):198-206.

32. Vinci DM. Effective nutrition support programs for college athletes. Int J Sport Nutr Exerc Metab. 1998; 8(3):308-20.

33. American Dietetic Association. Coordinate programs in dietetics (PC) 2000 [cited 2000 Mar 7]. Available from: <www.eatright.org.careers. $\mathrm{htm} / \mathrm{s}$.

34. Rockwell MS, Nickols-Richardson SM, Thye FW. Nutrition knowledge, opinions and practices of coaches and athletic trainers at a division I university. Int J Sport Nutr Exerc Metab. 2001; 11(2): 174-85.

35. Padua J. Técnicas de investigación aplicadas a las ciencias sociales. México: Fondo de Cultura Económica; 1987.

36. Mayntz R, Holm K, Hubner P. Introducción a los métodos de la sociologia empírica. Madrid: Alianza Editorial; 1975.

37. Huerta JA, Pérez ISG, Castellanos ARC. Desarrollo curricular por competencias profesionales integrales. 2004 [aceso en 2004 dec 10]. Disponible en: <http://educacion.jalisco.gob.mx/ consulta/educar/13/13Huerta.html>.

38. Santos LAS, Silva MCM, Santos JM, Assunção MP. Projeto pedagógico do programa de graduação em nutrição da Universidad Federal da Bahia: uma proposta em construção. Rev Nutr. 2005; 18(1): 105-17.

39. Santos LAS. Educação alimentar e nutricional no controle de praticas alimentares saudáveis. Rev Nutr. 2005; 18(5):681-92.

Recibido el: 10/4/2007

Versión final re-apresentada el: 10/12/2007

Aprobado el: 23/7/2008 